\title{
Anemia como predictor de severidad en la COVID-19: una revisión exploratoria
}

\section{Utility of anemia as a severity predictor in COVID-19: A scoping review}

Eduardo Tuta-Quintero ${ }^{1}$ https://orcid.org/0000-0002-7243-2238 Estefania Collazos https://orcid.org/0000-0003-1104-428X Juan Coronado-Sarmiento ${ }^{1}$ https://orcid.org/0000-0002-0470-9370 Juan Pimentel ${ }^{2}$ https://orcid.org/0000-0002-6842-3064

\begin{abstract}
Resumen
Introducción: La infección por SARS-CoV-2 con manifestaciones clínicas graves y críticas se ha asociado a descenso en los niveles plasmáticos de hemoglobina, siendo necesario comprender el impacto que tiene la anemia en la progresión a los desenlaces clínicos y mortalidad. Objetivo: Describir el estado actual de las publicaciones científicas hasta el 20 de junio de 2021, sobre la relación entre la anemia y la severidad clínica de la COVID-19, y su utilidad como factor pronóstico. Metodología: Revisión sistemática exploratoria en PubMed y Scopus, incluyendo estudios observacionales analíticos y descriptivos en inglés y español. Resultados: Se incluyeron un total de 14 publicaciones acumulando una población de 29755 pacientes: estudios de cohorte retrospectivo $(n=8)$, cohorte prospectivo $(n=3)$, estudio de casos y controles $(n=1)$, corte transversal $(n=1)$ y corte longitudinal $(n=1)$. El país de origen de los autores es en su gran mayoría China $(n=6)$, seguido de Estados Unidos $(n=2)$, Italia $(n=1)$, Grecia $(n=1)$, Etiopía $(n=1)$, Corea del Sur $(n=1)$, Irán $(n=1)$, India $(n=1)$, y Austria $(n=1)$. Conclusión: La anemia es prevalente en pacientes con infección SARS-CoV-2, principalmente en las presentaciones clínicas graves y críticas. Se asocia a estancias hospitalarias prolongadas, complicaciones y deterioro clínico de los pacientes.
\end{abstract}

Palabras clave: SARS-CoV-2; COVID-19; Anemia; Revisión sistemática exploratoria.

\begin{abstract}
Introduction: SARS-CoV2 infection with severe clinical manifestations has been associated with a decrease in plasma hemoglobin levels. This suggests the need to understand the impact that anemia has in the clinical outcomes of patients with COVID-19. Objective: Describe the extent and nature of the scientific publications assessing the relationship between anemia and COVID-19. Methodology: Scoping review including PubMed and Scopus, considering analytic and descriptive observational studies in English and Spanish. Result: 14 publications were found including 29755 patients: retrospective cohort $(n=8)$, prospective cohort $(n=3)$, case control studies $(n=1)$, cross-sectional $(n=1)$ and longitudinal case series $(n=1)$. The authors birthplace was China $(n=6)$, followed by the United States $(n=2)$, Italy $(n=1)$, Greece $(n=1)$, Ethiopia $(n=1)$, South Korea $(n=1)$, Iran $(n=1)$, India $(n=1)$ and Austria $(n=1)$. Conclusion: Anemia is prevalent in SARS-CoV-2 infected patients, mostly in critical clinical presentations. It is associated with long hospitalizations, clinical complications, and deterioration of patient's survival.
\end{abstract}

Key words: SARS-CoV-2; COVID-19; Anemia; Systematic review.

1. Facultad de medicina, Universidad de La Sabana, Chía, Colombia. 2. Departamento de Medicina Familiar y Salud Pública, Universidad de La Sabana, Chía, Colombia. Autor de correspondencia: Eduardotuqu@unisabana.edu.co

\section{Introducción}

La actual pandemia por la enfermedad del coronavirus 2019 (COVID-19) es causada por la infección por el coronavirus de tipo 2 causante del síndrome respiratorio agudo severo (SARS-CoV-2). Debido a su alta y rápida transmisibilidad por gotitas respiratorias y la escasa evidencia sobre variables clínicas y paraclínicas que determinen el curso de la enfermedad, este virus ha generado un gran impacto socioeconómico y en salud pública (Henry et al., 2020). Aproximadamente el $80 \%$ de los pacientes con infección por SARS-CoV-2 son asintomáticos, el $20 \%$ suelen presentar sintomatología clínica leve como fiebre, mialgia, fatiga y cefalea, y el $14 \%$ progresa a un estado grave dado por frecuencia respiratoria $\geq 30 \mathrm{rpm}$, saturación de oxígeno en sangre $\leq 93 \%$, una relación presión parcial de oxígeno y fracción de oxígeno inspirado ( $\mathrm{PaFiO} 2)<300$ y/o infiltrados pulmonares (Wu et al., 2020a, b). Sin embargo, el 6\% de las personas pueden desarrollar un estado crítico con síndrome de dificultad respiratoria aguda (SDRA), shock séptico y /o falla multiorgánica que requiere terapia en la unidad de cuidados intensivos (UCI) (Yang et al., 2020). Estos 
pacientes son generalmente adultos mayores con comorbilidades cardiovasculares y metabólicas (Henry et al., 2020; Yang et al., 2020: Wu et al., $2020 \mathrm{a}, \mathrm{b})$.

En Latinoamérica, la anemia tiene una alta prevalencia en la población, afectando principalmente niños menores de 6 años y mujeres, con rangos que varían desde el $4 \%$ en países como Costa Rica y Chile, alcanzando el $40 \%$ en países como Haití ${ }^{5}$, esto, sumado a los hallazgos de múltiples estudios, que al buscar algún factor pronóstico en la infección por SARS-COV2, han encontrado que los niveles de hemoglobina (Hb) varían en los pacientes de acuerdo a su supervivencia (sobrevivientes vs. fallecidos), o, de acuerdo con la severidad de la enfermedad (Wang et al., 2020). Recientes estudios sugieren que bajos niveles de hemoglobina $(\mathrm{Hb})$ en pacientes con COVID-19 pueden impactar negativamente el curso de la enfermedad. Se sugiere una modificación de la eritropoyesis debido a alteraciones en el metabolismo del hierro (Fan et al., 2020; Henry et al., 2020). Los procesos catabólicos acelerados asociados a un estado hiper inflamatorio como en la infección por SARS-CoV-2 pueden generar problemas en el metabolismo del hierro, y como consecuencia, procesos anémicos e hiperferritinemia (Ganz et al., 2020). La anemia reduce el suministro tisular de oxígeno, aumentando el riesgo de desarrollar SDRA y falla multiorgánica hiperferritinemia (Taneri et al., 2020). Comprender el impacto que tiene la anemia en los desenlaces clínicos en pacientes con COVID-19 permitirá desarrollar estrategias de manejo médico oportunas y eficaces. El objetivo de nuestra revisión fue explorar la evidencia médica disponible hasta el 20 de junio de 2021 sobre la relación entre la anemia como predictor de severidad de la COVID-19 y su utilidad clínica como factor pronóstico.

\section{Metodología}

La presente revisión sistemática exploratoria siguió los pasos propuestos por Arksey \& O'Malley, (2005) y Levac, (2010) así: (1.) identificar la pregunta de investigación, (2.) buscar los documentos relevantes, (3.) seleccionar los estudios, (4.) extraer los datos, y (5.) resumir y reportar los resultados. La revisión respondió a las preguntas: ¿Cuál es la literatura existente explorando la relación entre la anemia y el desarrollo de infección por SARS-CoV-2 con presentación clínica severa? ¿Cuál es la utilidad clínica de la anemia como predictor de desarrollar COVID-19 grave?

\section{Criterios de elegibilidad}

Los criterios de inclusión fueron: estudios observacionales analíticos; publicaciones evaluando el impacto de la anemia sobre la severidad de la COVID-19, o evaluando la anemia como factor pronóstico de severidad en la COVID19. Se consideraron publicaciones en inglés o español. Se excluyeron publicaciones que involucraran pacientes con anemias de carácter hereditario o autoinmunes. No se consideró un límite de fecha en nuestra búsqueda. La última actualización de la búsqueda fue junio 20 de 2021.

\section{Estrategia de búsqueda, selección de estudios y extracción de datos}

La búsqueda incluyó las bases de datos PubMed y SCOPUS. Se utilizaron operadores lógicos y palabras clave para cada sistema de datos. Utilizando la aplicación web de acceso gratuito para la gestión de revisiones sistemáticas Rayyan (Ouzzani et al., 2016), los autores revisaron y eligieron de forma independiente los títulos y resúmenes de las publicaciones candidatas. Se utilizó el debate y consenso para resolver las diferencias entre los investigadores. Posteriormente, fueron removidos los manuscritos duplicados y los documentos seleccionados fueron obtenidos en texto completo a través de la librería de la Universidad de la Sabana.

El siguiente paso fue la extracción de datos. Usando Microsoft Excel, un formato basado en las variables que responderían las preguntas de investigación fue creado. Este paso involucró reuniones regulares para discutir y ajustar los formatos. El método empleado fue el descriptivo-analítico (Arksey \& O'Malley, 2005) para recolectar información estándar de cada documento. Primero, se ajustó el formato usando $5 \%$ de los documentos para determinar si el método de extracción de datos era adecuado. A continuación, se extrajo la información de todos los documentos restantes. La extracción de la información, cuando estaba disponible, se realizó así: autores, tipo de documento (estudio clínico, revisión sistemática), características de la población, objetivo, fecha de publicación, revista, país de los autores, y principales hallazgos.

\section{Síntesis y presentación de los resultados}

La presentación de resultados de esta revisión sigue las categorías propuestas por Grudniewicz et al., (2016): (i) un resumen de las características y distribución de las publicaciones incluidas, y (ii) una síntesis narrativa de los resultados. En este artículo, seguimos la extensión PRISMA para reportar revisiones sistemáticas exploratorias 
(PRISMA-ScR) (Tricco et al., (2016). Se desarrolló una lista de chequeo, ejemplo de formato diligenciado (tabla 2). Este trabajo no requirió aprobación ética.

\section{Resultados}

Se incluyeron un total de 14 publicaciones (figura 1), en la que se encuentran estudios de cohorte retrospectivo $(n=8)$, cohorte prospectivo $(n=3)$, estudio de casos y controles $(n=1)$, corte transversal $(n=1)$ y corte longitudinal $(n=1)$. El país de origen de los autores es en su gran mayoría China $(n=6)$, seguido de Estados Unidos $(n=2)$, Italia $(n=1)$, Grecia $(n=1)$, Etiopía $(n=1)$, Corea del Sur $(n=1)$, Irán $(n=1)$, India $(n=1)$, y Austria $(n=1)$, con una población total evaluada de 29755 pacientes. Las principales características de estos documentos se encuentran en la tabla 1.

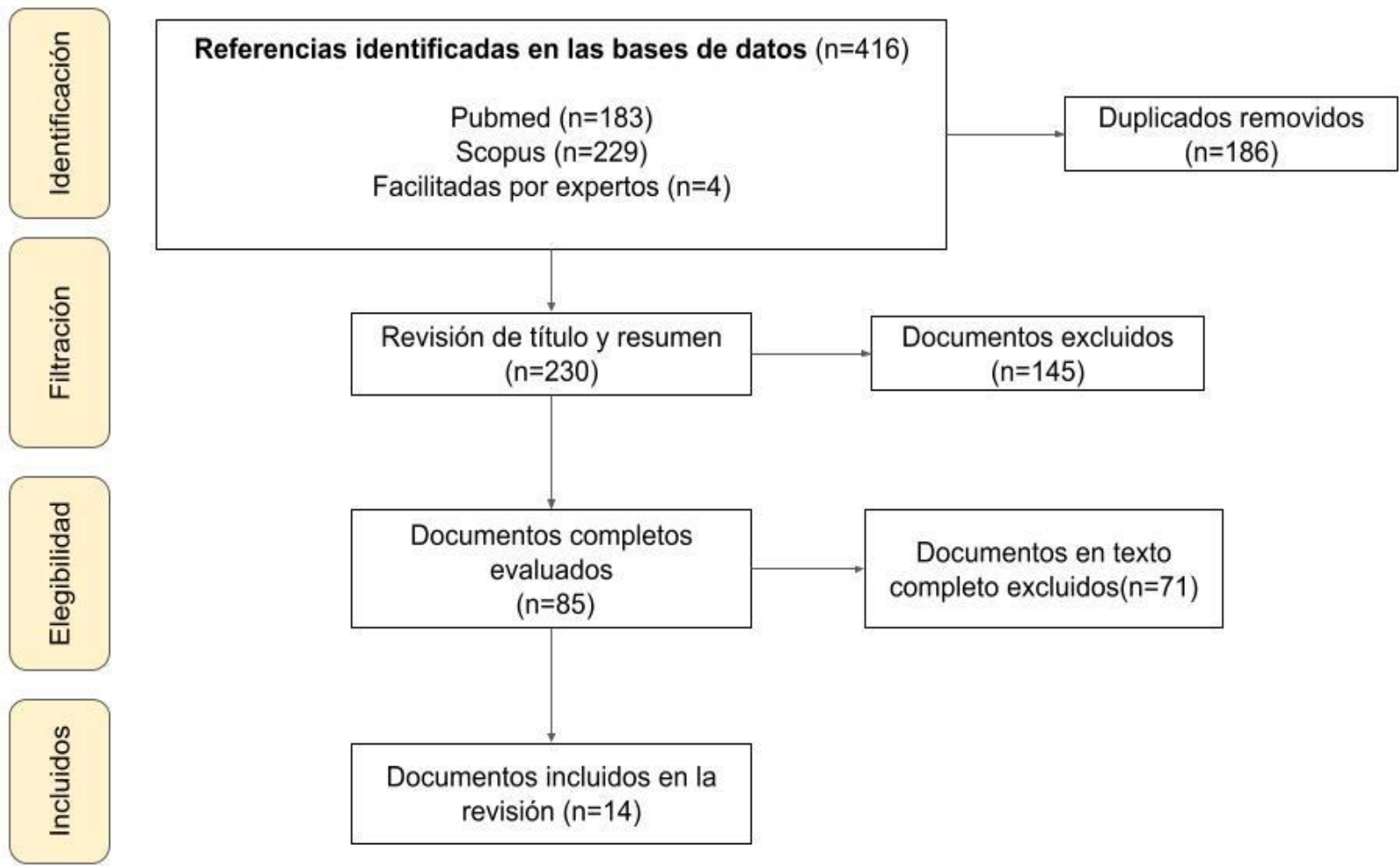

Figura 1. Revisión sistemática

\section{Cohorte retrospectiva}

Tao et al. (2021), evaluaron el impacto de la anemia en el curso clínico de 222 pacientes con diagnóstico de COVID-19. La anemia se definió de la siguiente manera: hemoglobina ( $\mathrm{Hb})$ de menos de $12 \mathrm{~g} / \mathrm{dL}$ en mujeres y menos de $13 \mathrm{~g} / \mathrm{dL}$ en hombres. La gravedad de la anemia se clasificó como leve de 11-11,9 g / dL para mujeres y 11-12,9 g / dL para hombres, moderada de 8-10,9 g / dL o severa menor de $8 \mathrm{~g} / \mathrm{dL}$. Un total de 79 (35,6\%) pacientes presentaron anemia, reportando una disminución de la $\mathrm{Hb}$ en los pacientes con enfermedades graves y críticas en comparación con pacientes leve o moderadamente enfermos por COVID-19 (32,2\% frente a 70,0\%, p =0,001).

Además, los pacientes con anemia eran adultos mayores y comórbidos con enfermedad renal crónica $(0,0 \%$ frente a 3,8\%), enfermedad cerebrovascular (3,5\% frente a 15,2\%) y enfermedad pulmonar obstructiva crónica (EPOC) $(0,0 \%$ frente a $10,1 \%)(p<0,005)$. Los niveles de la velocidad de sedimentación globular (VSG), dímero D, la fracción $\mathrm{N}$-terminal de los péptidos natriuréticos cerebrales (NT-proBNP) y nitrógeno ureico (BUN) en sangre fueron más altos en los pacientes anémicos $(p<0,005)$. La alteración en los niveles de $\mathrm{Hb}$ no se relacionó con un aumento en la mortalidad en las diferentes presentaciones clínicas de la infección por SARS-CoV-2 $(p=0,996)$. Los autores concluyeron que la anemia es un factor independiente para la severidad de la enfermedad por COVID-19, pese a no observarse una diferencia estadísticamente significativa en cuanto a la mortalidad. 
Tabla 1. Características de artículos explorados

\begin{tabular}{|c|c|c|c|c|c|c|c|}
\hline Autores & $\begin{array}{c}\text { Tipo de } \\
\text { documento }\end{array}$ & $\begin{array}{c}\text { Características de la } \\
\text { población }\end{array}$ & Objetivo & $\begin{array}{c}\text { Fecha de } \\
\text { publicación } \\
\text { (dd/mm/aaaa) }\end{array}$ & Revista & $\begin{array}{l}\text { País de } \\
\text { los } \\
\text { autores }\end{array}$ & Hallazgo principal / contribución \\
\hline Tao et al. & $\begin{array}{l}\text { Cohorte } \\
\text { retrospectiva }\end{array}$ & $\begin{array}{l}79 \text { pacientes con } \\
\text { anemia y } 143 \text { sin } \\
\text { anemia, con COVID - } \\
19 \text { confirmado }\end{array}$ & $\begin{array}{l}\text { Evaluar las características clínicas de los } \\
\text { pacientes con anemia y establecer la } \\
\text { relación de ésta con la severidad de la } \\
\text { COVID-19 }\end{array}$ & $19 / 08 / 2020$ & $\begin{array}{l}\text { Journal of } \\
\text { medical virology }\end{array}$ & China & $\begin{array}{l}\text { La anemia se asocia con una respuesta } \\
\text { inflamatoria más severa, y de hecho, es } \\
\text { un factor de riesgo independiente para } \\
\text { la severidad de la COVID-19 }\end{array}$ \\
\hline Oh et al. & $\begin{array}{l}\text { Cohorte } \\
\text { retrospectiva }\end{array}$ & $\begin{array}{l}733 \text { pacientes, }>18 \\
\text { años, con COVID-19 } \\
\text { confirmado }\end{array}$ & $\begin{array}{l}\text { Valorar las características clínicas de los } \\
\text { pacientes con COVID-19 grave }\end{array}$ & $27 / 03 / 2021$ & $\begin{array}{l}\text { American } \\
\text { Journal of } \\
\text { Emergency } \\
\text { Medicine }\end{array}$ & $\begin{array}{l}\text { Estados } \\
\text { Unidos }\end{array}$ & $\begin{array}{l}\text { La anemia se encontró como un factor } \\
\text { independiente de mortalidad por todas } \\
\text { las causas en pacientes hospitalizados } \\
\text { con COVID-19 }\end{array}$ \\
\hline $\begin{array}{l}\text { Bergamaschi } \\
\quad \text { et al. }\end{array}$ & $\begin{array}{l}\text { Cohorte } \\
\text { prospectivo }\end{array}$ & $\begin{array}{l}277 \text { pacientes con una } \\
\text { media de } 71 \text { años, } 206 \\
\text { con COVID-19 } \\
\text { confirmado }\end{array}$ & $\begin{array}{l}\text { Estudiar la prevalencia de anemia en los } \\
\text { pacientes con COVID-19, y su impacto } \\
\text { clínico durante la hospitalización }\end{array}$ & 08/01/2021 & $\begin{array}{l}\text { Clinical and } \\
\text { experimental } \\
\text { medicine }\end{array}$ & Italia & $\begin{array}{l}\text { En pacientes que presentaban anemia, el } \\
\text { ADE se encontró como factor } \\
\text { independiente para mortalidad. }\end{array}$ \\
\hline Gupta et al. & $\begin{array}{l}\text { Cohorte } \\
\text { prospectivo }\end{array}$ & $\begin{array}{l}200 \text { pacientes, con } \\
\text { edad de 40+/-17 años, } \\
\text { con COVID-19 } \\
\text { confirmado }\end{array}$ & $\begin{array}{c}\text { Evaluar el perfil clínico y paraclínico de } \\
\text { los pacientes para establecer un pronóstico } \\
\text { y resultado clínico }\end{array}$ & $09 / 11 / 220$ & $\begin{array}{l}\text { Monaldi } \\
\text { Archives for } \\
\text { Chest Disease }\end{array}$ & India & $\begin{array}{l}\text { Los pacientes que presentaron anemia, } \\
\text { tuvieron mayor ingreso a UCI }\end{array}$ \\
\hline Ji et al. & $\begin{array}{l}\text { Casos y } \\
\text { controles }\end{array}$ & $\begin{array}{l}7341 \text { pacientes, } \\
\text { mayores de } 18 \text { años, } \\
\text { con COVID-19 } \\
\text { confirmado }\end{array}$ & $\begin{array}{l}\text { Evaluar el impacto de la infección y } \\
\text { comorbilidades en pacientes con COVID- } \\
\text { 19, para establecer el resultado clínico }\end{array}$ & $29 / 06 / 2020$ & $\begin{array}{l}\text { Journal of } \\
\text { Korean Medical } \\
\text { Science }\end{array}$ & $\begin{array}{l}\text { Corea } \\
\text { del Sur }\end{array}$ & $\begin{array}{l}\text { Los pacientes que presentaron anemia, } \\
\text { tuvieron un riesgo disminuido de } \\
\text { presentar COVID-19 }\end{array}$ \\
\hline Zhao et al. & $\begin{array}{l}\text { Cohorte } \\
\text { retrospectiva }\end{array}$ & $\begin{array}{l}29 \text { pacientes, mayores } \\
\text { de } 18 \text { años, con } \\
\text { COVID-19 } \\
\text { confirmado }\end{array}$ & $\begin{array}{l}\text { Evaluar los aspectos clínicos y } \\
\text { epidemiológicos de los pacientes que } \\
\text { cursan con COVID-19 }\end{array}$ & $05 / 06 / 2020$ & $\begin{array}{l}\text { Frontiers in } \\
\text { Medicine }\end{array}$ & China & $\begin{array}{l}\text { La anemia, la elevación del dímero-D, } \\
\text { de la IL-6 o una disminución en el } \\
\text { calcio o la albúmina se asociaron con } \\
\text { una enfermedad más severa }\end{array}$ \\
\hline $\begin{array}{l}\text { Bellmann- } \\
\text { Weiler } \text { et al. }\end{array}$ & $\begin{array}{l}\text { Cohorte } \\
\text { retrospectiva }\end{array}$ & $\begin{array}{l}259 \text { pacientes, } \\
\text { mayores de } 18 \text { años, } \\
\text { con COVID-19 } \\
\text { confirmado }\end{array}$ & $\begin{array}{c}\text { Evaluar la presencia de anemia y de } \\
\text { homeostasis del hierro en pacientes con } \\
\text { COVID-19 }\end{array}$ & $29 / 07 / 2020$ & $\begin{array}{l}\text { Journal of } \\
\text { Clinical } \\
\text { Medicine }\end{array}$ & Austria & $\begin{array}{l}\text { La anemia de carácter moderado y } \\
\text { severo se asociaron con mayor } \\
\text { mortalidad, pero no hubo relación con } \\
\text { ingreso a la UCI o con la necesidad de } \\
\text { ventilación. }\end{array}$ \\
\hline Xu et al. & $\begin{array}{l}\text { Cohorte } \\
\text { retrospectiva }\end{array}$ & $\begin{array}{l}703 \text { pacientes, } \\
\text { mayores de } 18 \text { años } \\
\text { con COVID-19 } \\
\text { confirmado }\end{array}$ & $\begin{array}{c}\text { Evaluar la mortalidad por todas las causas } \\
\text { en pacientes ingresados a hospitalización } \\
\text { y/o UCI con COVID-19 }\end{array}$ & $15 / 05 / 2020$ & Theranostics & China & $\begin{array}{c}\text { Pacientes con anemia, tuvieron una } \\
\text { enfermedad más severa y mayor ingreso } \\
\text { a UCI }\end{array}$ \\
\hline Zhang et al. & $\begin{array}{l}\text { Cohorte } \\
\text { retrospectiva }\end{array}$ & $\begin{array}{l}663 \text { pacientes, } \\
\text { mayores de } 18 \text { años } \\
\text { con COVID-19 } \\
\text { confirmado }\end{array}$ & $\begin{array}{l}\text { Analizar los factores clínicos que } \\
\text { conllevan a una enfermedad por COVID- } \\
19 \text { más severa, que limitan la mejoría } \\
\text { clínica y factores de mortalidad }\end{array}$ & $15 / 04 / 2020$ & $\begin{array}{l}\text { Clinical } \\
\text { Microbiology } \\
\text { and Infection }\end{array}$ & China & $\begin{array}{l}\text { La anemia se relacionó con un peor } \\
\text { pronóstico de la COVID-19, incluyendo } \\
\text { enfermedad más severa e ingreso a UCI }\end{array}$ \\
\hline Zheng et al. & $\begin{array}{l}\text { Cohorte } \\
\text { retrospectiva }\end{array}$ & $\begin{array}{l}161 \text { pacientes, } \\
\text { mayores de } 18 \text { años, } \\
\text { edad media de } 45 \\
\text { años, con COVID-19 } \\
\text { confirmado }\end{array}$ & $\begin{array}{l}\text { Investigar las características clínicas de los } \\
\text { pacientes con COVID-19 }\end{array}$ & $24 / 03 / 2020$ & $\begin{array}{l}\text { European } \\
\text { Review for } \\
\text { Medical and } \\
\text { Pharmacological } \\
\text { Sciences }\end{array}$ & China & $\begin{array}{l}\text { No hubo diferencia estadística entre } \\
\text { pacientes con o sin anemia como factor } \\
\text { de severidad. La edad, hipertensión, el } \\
\text { requerimiento de oxígeno, los niveles } \\
\text { de lactato sérico y la linfopenia fueron } \\
\text { factores de peor pronóstico }\end{array}$ \\
\hline $\begin{array}{l}\text { Dinevari et } \\
\quad \text { al. }\end{array}$ & $\begin{array}{l}\text { Cohorte } \\
\text { prospectiva }\end{array}$ & $\begin{array}{l}1274 \text { pacientes, } \\
\text { mayores de } 18 \text { años, } \\
\text { edad media de } \\
\text { 64.4años, con } \\
\text { COVID-19 } \\
\text { confirmado }\end{array}$ & $\begin{array}{c}\text { Evaluar la asociación de anemia en } \\
\text { pacientes con COVID-19, con base en su } \\
\text { supervivencia }\end{array}$ & $27 / 04 / 2021$ & $\begin{array}{l}\text { BMC Infectious } \\
\text { Diseases }\end{array}$ & Irán & $\begin{array}{l}\text { La presencia de anemia se asoció con } \\
\text { peores desenlaces clínicos y con mayor } \\
\text { severidad de la enfermedad }\end{array}$ \\
\hline Estiri et al. & $\begin{array}{c}\text { Corte } \\
\text { longitudinal }\end{array}$ & $\begin{array}{l}16709 \text { registros } \\
\text { médicos de pacientes } \\
\text { con COVID-19 } \\
\text { confirmado }\end{array}$ & $\begin{array}{l}\text { Evaluar la asociación entre } 46 \text { condiciones } \\
\text { clínicas y el resultado clínico de la } \\
\text { COVID-19 }\end{array}$ & 04/02/2021 & Nature & $\begin{array}{l}\text { Estados } \\
\text { Unidos }\end{array}$ & $\begin{array}{l}\text { La anemia se asoció con mayor } \\
\text { mortalidad, sin embargo la edad es la } \\
\text { variable más significativa para predecir } \\
\text { severidad de enfermedad. }\end{array}$ \\
\hline Mao et al. & $\begin{array}{l}\text { Cohorte } \\
\text { retrospectiva }\end{array}$ & $\begin{array}{l}127 \text { pacientes, } \\
\text { mayores de } 18 \text { años, } \\
\text { edad media de } \\
\text { 64.4años, con } \\
\text { COVID-19 } \\
\text { confirmado }\end{array}$ & $\begin{array}{l}\text { Explorar las manifestaciones } \\
\text { hematológicas en pacientes recuperados de } \\
\text { COVID- } 19\end{array}$ & $01 / 02 / 2021$ & $\begin{array}{l}\text { Annals of } \\
\text { Hematology }\end{array}$ & China & $\begin{array}{l}\text { Los pacientes que presentaron anemia, } \\
\text { linfopenia, eosinopenia y monocitosis } \\
\text { tuvieron una enfermedad más severa }\end{array}$ \\
\hline Araya et al. & $\begin{array}{l}\text { Corte } \\
\text { transversal }\end{array}$ & $\begin{array}{l}334 \text { pacientes, } \\
\text { mayores de } 18 \text { años, } \\
\text { edad media de } \\
\text { 49.4años, con } \\
\text { COVID-19 } \\
\text { confirmado }\end{array}$ & $\begin{array}{l}\text { Determinar la magnitud de las } \\
\text { anormalidad hematológicas en pacientes } \\
\text { con COVID-19 }\end{array}$ & $02 / 03 / 2021$ & $\begin{array}{l}\text { Journal of } \\
\text { Multidisciplinary } \\
\text { Healthcare }\end{array}$ & Etiopía & $\begin{array}{l}\text { Los pacientes con COVID-19 severo } \\
\text { desarrollaron anemia y leucocitosis a } \\
\text { expensas de neutrofilia }\end{array}$ \\
\hline
\end{tabular}

Notas: LLC: leucemia linfoide crónica, ADE: Ancho de distribución eritrocitaria, UCI: Unidad de cuidados intensivos, IL: interleucinas. 
Zhang et al. (2020), evaluaron retrospectivamente una cohorte de 663 pacientes con COVID-19, confirmados mediante PCR. El estudio proporcionó características clínicas y factores asociados con la gravedad y la mortalidad de la enfermedad. Un total de 247 tenían al menos una enfermedad crónica, comprometiendo más frecuentemente el sistema cardiovascular $(21,1 \%)$, endocrino $(10,1 \%)$ y respiratorio $(7,9 \%)$. El $23.1 \%$ de los pacientes en quienes se documentó anemia durante la hospitalización desarrolló una enfermedad leve a moderada, un $43.6 \%$ presentó enfermedad severa, y un $37.2 \%$ presentó un estado crítico $(p<0.001)$. De la misma manera, los pacientes que presentaron anemia tuvieron una evolución tórpida, y sin mejoría en un $39.7 \%$ ( $p=0.006)$, con una mayor tasa de ingreso a la UCI y mortalidad (44.4\%, $p=0,414)$.

Bellmann-Weiler et al. (2020), evaluaron el impacto de la anemia y de la homeostasis del hierro en 259 pacientes hospitalizados con infección por COVID-19, y su relación con la severidad de la enfermedad. En el análisis retrospectivo, 32 pacientes fallecieron y 53 pacientes fueron trasladados a la UCI, con un requerimiento de ventilación mecánica invasiva (VMI) para 35 de estos pacientes. Se documentó una prevalencia relativamente alta de anemia en pacientes con COVID-19 (24.7\%), destacando que estos pacientes tenían una edad más avanzada, función renal disminuida y valores elevados de PCR. La causa de la anemia fue en un $68.8 \%$ de origen inflamatorio asociada a la infección por SARS-CoV-2 junto a una mayor mortalidad (OR:3.729; IC del 95\%: 1.739-7.995; $p=0.001$ ) frente al grupo sin anemia. Sin embargo, la anemia no se asoció con un mayor ingreso a la UCI (OR:0.867; IC del 95\%: 0.4241.774; $\mathrm{p}=0.696$ ) o requerimiento de VMI (OR: 1.259; IC del 95\%: 0.569-2.788; $\mathrm{p}=0.570$ ). Los autores concluyeron que la anemia es prevalente en pacientes hospitalizados por COVID-19 y se asocia con un aumento de la mortalidad.

\section{Cohorte prospectiva}

Dinevari et al. (2021), investigaron la asociación prospectiva entre la anemia y los desenlaces de la COVID-19 en 1274 pacientes hospitalizados en Tabriz, Irán. Encontraron que el 48.2\% de los pacientes que ingresaron al hospital se encontraban anémicos, principalmente en población mayor de 65 años $(p=0.02)$, hombres $(\mathrm{p}=0.03)$ y con comorbilidades como la hipertensión arterial esencial $(\mathrm{p}<0.001)$, diabetes mellitus $(\mathrm{p}<0.001)$, enfermedad renal $(\mathrm{p}<0.001)$ y con cáncer $(\mathrm{p}<0.001)$. Además, la anemia fue determinante en el ingreso a la UCI $(20.6 \%$ en pacientes no anémicos vs. $35.93 \%$ en pacientes anémicos $(\mathrm{p}<0.001)$ ) y la necesidad de VMI $(14.8 \%$ en pacientes no anémicos vs. $27.8 \%$ en pacientes anémicos $(\mathrm{p}<0.001))$. Los autores determinaron que la anemia era un factor independiente de severidad para COVID-19, reportando una mortalidad del $23.9 \%$ en paciente anémico frente a un $13.8 \%$ sin alteración de la $\mathrm{Hb}(\mathrm{p}<0.001)$.

\section{Casos y controles}

Ji et al. (2020), mediante un estudio retrospectivo de casos y controles, evaluaron el impacto de las comorbilidades sobre el riesgo de infección por SARS-CoV-2 y presentación clínica de la enfermedad en 7.341 pacientes frente a 212.620 controles. En el grupo control, un total de 954 pacientes presentaron enfermedad grave por COVID-19, de los cuales 140/954 (14,7) fueron diagnosticados con anemia a lo largo de su enfermedad frente a 365/6,387 (5,7\%) pacientes con enfermedad moderada o leve ( $\mathrm{p}<0,001)$. La hipertensión arterial (OR: 1.245-1.317) y la enfermedad renal crónica (OR: 2.052-2.178) se asociaron con un mayor riesgo de COVID-19 grave, a diferencia del Alzheimer o enfermedades neurodegenerativas (OR: 0,693-0,701).

\section{Corte longitudinal}

En un estudio de cohorte retrospectiva, Estiri et al. (2021), analizaron los registros médicos de 16.709 pacientes con COVID-19 confirmado por PCR, con el fin de crear un modelo predictivo para la mortalidad por COVID19. Utilizaron 46 variables clínicas y 5 demográficas, dentro de las cuales estudiaron la anemia. Un total de 15.879 pacientes sobrevivieron a la infección y 830 murieron. El 58.9\% los pacientes que no sobrevivieron a la COVID-19 presentaban anemia, asociándose a un peor pronóstico clínico (OR 3.64; p<0.01). Sin embargo, la variable más importante documentada en este estudio fue la edad, pues la severidad de la enfermedad por COVID-19 aumentó a edades avanzadas (pacientes entre 65-85 años (OR:4.3; p<0.01), y mayores de 85 años (OR:11.3; p<0.01). En pacientes mayores de 65 las enfermedades que comprometen el sistema pulmonar como el cáncer y la enfermedad pulmonar obstructiva crónica (EPOC) fueron importantes predictores de mortalidad.

\section{Corte transversal}

Araya et al. (2021), determinaron la utilidad clínica de las alteraciones en las líneas sanguíneas en 334 pacientes con COVID-19 ingresados a un centro de salud en Etiopía. El 69,8\% y 12,3\% de los pacientes presentaba enfermedad moderada y crítica por COVID-19, respectivamente. Las citopenias, entre ellas la anemia, fue mayor en pacientes graves $(43,3 \%)(p=0,06)$ y críticos $(46,3 \%)(p=0,44)$ que en pacientes con enfermedad moderada $(39,5 \%)$. La anemia fue diagnosticada en el $24,9 \%$, de los cuales el 1,2\% de los pacientes presentaban anemia grave $(<8 \mathrm{~g} / \mathrm{dL})$, y su probabilidad de desarrollar anemia fue 2,35 veces mayor en pacientes con enfermedad grave que moderada ( $p=$ $0,007)$, en hombres $(26,4 \%)$ con edades entre los 18 a 35 años $(28,4 \%)$. Los autores concluyeron que las alteraciones 
hematológicas como la anemia, neutrofilia/linfopenia, leucocitosis y neutrofilia se asocian con un posible deterioro en el curso clínico de la COVID-19.

\section{Discusión}

Esta revisión de la literatura exploró la literatura científica explorando la relación entre la presentación de anemia en pacientes con COVID-19, el curso clínico de la enfermedad, y su utilidad clínica como factor pronóstico. La anemia es prevalente en pacientes con infección SARS-CoV-2. Se asocia a presentaciones clínicas graves y críticas, estancias hospitalarias prolongadas, y un deterioro en la supervivencia de los pacientes como lo citan Ji et al., (2020); Zhang et al., (2020); Araya et al., (2021); Bellmann-Weiler et al., (2021); Estiri et al., (2021) y Oh et al., (2021) VERIFICAR LOS RESALYTADOS EN AMARILLO. Además, la gravedad de la anemia se asoció con elevación de marcadores inflamatorios o de disfunción orgánica como la VSG, el dímero-D, NT-proBNP y BUN (Tao et al., 2021). Las enfermedades crónicas con compromiso de los sistemas cardiovasculares, metabólico y respiratorio fueron tres enfermedades coexisten con la anemia (Bellmann-Weiler et al., 2021 y Ji et al., 2020). Estos resultados indican que la anemia juega un papel importante en la fisiopatología del deterioro clínico de los pacientes con infección por SARS CoV-2, aumentando la tasa de complicaciones y morbimortalidad (Gupta et al., 2020 y Mao et al., 2021).

La exploración de la anemia como factor predictor de diversas patologías infecciosas ha ido ganando fuerza en los últimos años. En pacientes con neumonía bacteriana (Mujica-Coopman et al., 2015), es considerada un factor independiente de mortalidad a 90 días, asociándose con el desarrollo de lesión renal aguda, y deterioro de la función respiratoria (Se reporta un aumento de hasta un $36 \%$ en el puntaje Sequential Organ Failure Assessment por gramo de $\mathrm{Hb}$ por debajo de $10 \mathrm{gr} / \mathrm{dL}$ (Han et al., 2015). Así mismo, la asociación entre las alteraciones en las líneas celulares sanguíneas y la infección por SARS-CoV-2 depende de factores como la edad, sexo, comorbilidades y estado inmunológico, los cuales determinan en gran medida el curso clínico y mortalidad de la enfermedad por COVID-19. Pese a que existe diferencia con respecto a la metodología y características de la población entre los estudios, el porcentaje de anemia varía desde el 24,7\% al $48 \%$ de pacientes con diagnóstico de COVID-19 (Araya et al., 2021); Bellmann-Weiler et al., 2021 y Faghih Dinevari et al., 2021). La anemia puede presentarse junto a trombocitopenia y neutrofilia en pacientes mayores de 56 años (Araya et al., 2021). En este sentido, la asociación entre anemia y la gravedad o mortalidad del COVID-19 predispone más a los pacientes a desarrollar desenlaces clínicos negativos como la necesidad de VMI en UCI y muerte en pacientes con enfermedad grave por COVID-19 (Guan Guan et al., 2020 y Young et al., 2020).

Si bien, la prevalencia de la anemia, especialmente en países en vía de desarrollo5, corresponde a una de origen ferropénico por baja ingesta alimentaria, es la anemia asociada a la inflamación sistémica es considerada la más prevalente a nivel mundial y equivale al $40 \%$ de todas las anemias. Se relaciona con infecciones con estados de hiper inflamación sostenida. El desbalance inmunológico genera una deficiencia en los niveles de hierro plasmáticos debido a la retención de este en los macrófagos. Esta retención es mediada por citocinas inflamatorias como el factor de necrosis tumoral $\alpha$ (TNF- $\alpha$ ) e interleucinas 6 y 10 (IL-6, IL-10), junto a una inhibición de la absorción duodenal debido al aumento de las concentraciones de hepcidina, provocando una disminución en la eritropoyesis y síntesis del grupo hemo y $\mathrm{Hb}$ (Weiss et al., 2019). Un número importante de estudios han revelado el impacto sobre la Hb y el metabolismo del hierro de la infección por SARS-CoV-2, principalmente en sus presentaciones clínicas graves y el compromiso multiorgánico ${ }^{29}$. Niveles elevados de ferritina, valores normales de transferrina y valores disminuidos de hierro, son características clásicas de la anemia de origen inflamatorio en pacientes con COVID-19 (Hemauer et al., 2010 y Algassim et al., 2021).

La alteración del metabolismo del hierro disminuye la capacidad del sistema inmune para reprimir la replicación viral (Weiss et al., 2019). Además, cambios morfológicos de los glóbulos rojos debido a la destrucción por parte de la inmunidad innata y adaptativa celular, generan un aumento en la necesidad de transfusión, estancia hospitalaria y posiblemente complicaciones asociadas al síndrome de activación de macrófagos, severidad del síndrome de respuesta inflamatoria y de la tormenta de citoquinas (Capes et al., 2020). Por otro lado, la anemia potencia la activación del sistema nervioso simpático, llevando a un estado de inestabilidad hemodinámica dada por taquicardia, hipertensión arterial sistémica y un aumento de la tensión a nivel de la membrana alveolo capilar, ocasionando fuga capilar pulmonar y empeoramiento del SDRA en estos pacientes (Faghih Dinevari et al., 2020).

El diagnóstico y estratificación de la severidad COVID-19 de manera oportuna son fundamentales para prevenir resultados clínicos desfavorables (Lagadinou et al., 2020 y Tuta-Quintero et al., 2021). Los marcadores hematológicos anormales como anemia, linfopenia, trombocitopenia y neutrofilia se han asociado con la progresión y gravedad de la enfermedad, por lo cual su utilidad clínica cobra relevancia en unidades de urgencias y UCI para orientar el plan terapéutico de estos pacientes ${ }^{16,35}$. Si bien existen diferencias en las edades y comorbilidades de los pacientes, marcadores evaluados y parámetros diagnóstico establecidos entre los estudios, existe la necesidad de continuar explorando la relevancia clínica de estas variables. 


\section{Conclusiones}

La anemia es una condición prevalente en los pacientes con COVID-19 grave y crítica. Puede servir como predictor clínico del curso de la enfermedad, siendo importante control paraclínico con $\mathrm{Hb}$ y otros marcadores en pacientes hospitalizados con signos temprano de falla ventilatoria. Es necesario continuar explorando este fenómeno con nuevos estudios prospectivos con un mayor tamaño de muestra y periodo de seguimiento.

\section{Conflictos de interés}

Los autores declaran no tener conflictos de interés en la publicación de este artículo.

\section{Agradecimiento}

Ninguno

\section{Referencias}

Algassim, A.A., Elghazaly, A.A., Alnahdi, A.S., Mohammed-Rahim, O.M., Alanazi, A.G., Aldhuwayhi, N.A., Alanazi, M.M., Almutairi, M.F., Aldeailej, I.M., Kamli, N.A. \& Aljurf, M.D. (2021). Prognostic significance of hemoglobin level and autoimmune hemolytic anemia in SARS-CoV-2 infection. Ann Hematol, 100(1):37-43. https://doi.org/10.1007/s00277-020-04256-3

Araya, S., Wordofa, M., Mamo, M.A., Tsegay, Y.G., Hordofa, A., Negesso, A.E., Fasil, T., Berhanu, B., Begashaw, H., Atlaw, A., Niguse, T., Cheru, M. \& Tamir, Z. (2021). The Magnitude of Hematological Abnormalities Among COVID-19 Patients in Addis Ababa, Ethiopia. J Multidiscip Healthc, 2;14:545-554. https://doi.org/10.2147/JMDH.S295432

Arksey H, O’Malley L: Scoping studies: Towards a Methodological Framework. Int J Soc Res Methodol 2005, 8:19-32. Bellmann-Weiler, R., Lanser, L., Barket, R., Rangger, L., Schapfl, A., Schaber, M., Fritsche, G., Wöll, E., \& Weiss, G. (2020). Prevalence and Predictive Value of Anemia and Dysregulated Iron Homeostasis in Patients with COVID-19 Infection. Journal of clinical medicine, 9(8): 2429. https://doi.org/10.3390/jcm9082429

Capes, A., Bailly, S., Hantson, P., Gerard, L., \& Laterre, P. F. (2020). COVID-19 infection associated with autoimmune hemolytic anemia. Annals of hematology, 99(7): 1679-1680. https://doi.org/10.1007/s00277-020-04137-9

Estiri, H., Strasser, Z. H., Klann, J. G., Naseri, P., Wagholikar, K. B., \& Murphy, S. N. (2021). Predicting COVID-19 mortality with electronic medical records. NPJ digital medicine, 4(1): 15. https://doi.org/10.1038/s41746-021$\underline{00383-\mathrm{x}}$

Faghih Dinevari, M., Somi, M. H., Sadeghi Majd, E., Abbasalizad Farhangi, M., \& Nikniaz, Z. (2021). Anemia predicts poor outcomes of COVID-19 in hospitalized patients: a prospective study in Iran. BMC infectious diseases, 21(1): 170. https://doi.org/10.1186/s12879-021-05868-4

Fan, B. E., Chong, V., Chan, S., Lim, G. H., Lim, K., Tan, G. B., Mucheli, S. S., Kuperan, P., \& Ong, K. H. (2020). Hematologic parameters in patients with COVID-19 infection. American journal of hematology, 95(6): E131E134. https://doi.org/10.1002/ajh.25774

Ganz T. (2019). Anemia of Inflammation. The New England journal of medicine, 381(12): 1148-1157. https://doi.org/10.1056/NEJMra1804281

Grudniewicz, A., Nelson, M., Kuluski, K., Lui, V., Cunningham, H. V., X Nie, J., Colquhoun, H., Wodchis, W. P., Taylor, S., Loganathan, M., \& Upshur, R. E. (2016). Treatment goal setting for complex patients: protocol for a scoping review. BMJ open, 6(5):e011869. https://doi.org/10.1136/bmjopen-2016-011869

Guan, W. J., Ni, Z. Y., Hu, Y., Liang, W. H., Ou, C. Q., He, J. X., Liu, L., Shan, H., Lei, C. L., Hui, D., Du, B., Li, L. J., Zeng, G., Yuen, K. Y., Chen, R. C., Tang, C. L., Wang, T., Chen, P. Y., Xiang, J. \& Li, S. Y. (2020). Clinical Characteristics of Coronavirus Disease 2019 in China. The New England journal of medicine, 382(18): 1708-1720. https://doi.org/10.1056/NEJMoa2002032

Gupta, N., Ish, P., Kumar, R., Dev, N., Yadav, S. R., Malhotra, N., Agrawal, S., Gaind, R., Sachdeva, H., \& Covid Working Group, O. (2020). Evaluation of the clinical profile, laboratory parameters and outcome of two hundred COVID-19 patients from a tertiary centre in India. Monaldi archives for chest disease = Archivio Monaldi per le malattie del torace, 90(4): 10.4081. https://doi.org/10.4081/monaldi.2020.1507 
Han, S. S., Baek, S. H., Ahn, S. Y., Chin, H. J., Na, K. Y., Chae, D. W., \& Kim, S. (2015). Anemia Is a Risk Factor for Acute Kidney Injury and Long-Term Mortality in Critically Ill Patients. The Tohoku journal of experimental medicine, 237(4): 287-295. https://doi.org/10.1620/tjem.237.287

Hemauer, S.J., Kingeter, A.J., Han, X., Shotwell, M.S., Pandharipande, P.P. \& Weavind, L.M .(2017). Hemoglobina más baja diaria y riesgo de disfunción orgánica en pacientes críticamente enfermos. Medicina de cuidados intensivos , 45 (5): e479 - e484. https://doi.org/10.1097/CCM.0000000000002288

Henry, B. M., de Oliveira, M., Benoit, S., Plebani, M., \& Lippi, G. (2020). Hematologic, biochemical and immune biomarker abnormalities associated with severe illness and mortality in coronavirus disease 2019 (COVID-19): a meta-analysis. Clinical chemistry and laboratory medicine, 58(7): 1021-1028. https://doi.org/10.1515/cclm$\underline{2020-0369}$

Ji, W., Huh, K., Kang, M., Hong, J., Bae, G.H., Lee, R., Na, Y., Choi, H., Gong, S.Y., Choi, Y.H., Ko, K.P , Im, J.S. \& Jung, J. (2020). Efecto de las comorbilidades subyacentes sobre la infección y la gravedad de COVID-19 en Corea: un estudio de casos y controles a nivel nacional. Revista de ciencia médica coreana , 35 (25): e237. https://doi.org/10.3346/jkms.2020.35.e237

Lagadinou, M., Salomou, E. E., Zareifopoulos, N., Marangos, M., Gogos, C., \& Velissaris, D. (2020). Prognosis of COVID-19: Changes in laboratory parameters. Le infezioni in medicina, 28(suppl 1):89-95.

Levac, D., Colquhoun, H. \& O'Brien, K.K. (2010). Estudios de alcance: avance de la metodología. Implementation Sci 5: 69 https://doi.org/10.1186/1748-5908-5-69

Mao, J., Dai, R., Du, R. C., Zhu, Y., Shui, L. P., \& Luo, X. H. (2021). Hematologic changes predict clinical outcome in recovered patients with COVID-19. Annals of hematology, 100(3): 675-689. https://doi.org/10.1007/s00277$\underline{021-04426-\mathrm{x}}$

Mujica-Coopman, M. F., Brito, A., López de Romaña, D., Ríos-Castillo, I., Coris, H., \& Olivares, M. (2015). Prevalence of Anemia in Latin America and the Caribbean. Food and nutrition bulletin, 36(2 Suppl):S119 S128. https://doi.org/10.1177/0379572115585775

Oh, S.M., Skendelas, J.P., Macdonald, E., Bergamini, M., Goel, S. Choi, J., Segal, K.R., Vivek, K., Nair, S. \& Leff, J. (2021). La anemia al ingreso predice la mortalidad en pacientes con COVID-19: un estudio de cohorte retrospectivo de un solo centro. Revista estadounidense de medicina de emergencia, $48: 140$ 147. https://doi.org/10.1016/j.ajem.2021.03.083

Ouzzani, M., Hammady, H,, Fedorowicz, Z. \& Elmagarmid, A. (2016). Rayyan: una aplicación web y móvil para revisiones sistemáticas. Syst Rev 5, 210 https://doi.org/10.1186/s13643-016-0384-4

Taneri, P. E., Gómez-Ochoa, S. A., Llanaj, E., Raguindin, P. F., Rojas, L. Z., Roa-Díaz, Z. M., Salvador, D., Jr, Groothof, D., Minder, B., Kopp-Heim, D., Hautz, W. E., Eisenga, M. F., Franco, O. H., Glisic, M., \& Muka, T. (2020). Anemia and iron metabolism in COVID-19: a systematic review and meta-analysis. European journal of epidemiology, 35(8):763-773. https://doi.org/10.1007/s10654-020-00678-5

Tao, Z., Xu, J., Chen, W., Yang, Z., Xu, X., Liu, L., Chen, R., Xie, J., Liu, M., Wu, J., Wang, H., \& Liu, J. (2021). Anemia is associated with severe illness in COVID-19: A retrospective cohort study. Journal of medical virology, 93(3): 1478-1488. https://doi.org/10.1002/jmv.26444

Tricco, A,C,, Lillie, E, Zarin, W., O’Brien, K.K., Colquhoun, H, \& Levac, D. (2018). PRISMA Extension for Scoping Reviews (PRISMA-ScR): Checklist and Explanation. Ann Intern Med. 169:467. https://doi.org/10.7326/M18$\underline{0850}$

Tuta-Quintero, E., Vega-Corredor, M. C., Perdomo-Rodríguez, L. S., \& Pimentel, J. (2021). Colchicina, perspectivas de un viejo amigo para la reumatología en la COVID-19: una revisión exploratoria. Revista Colombiana de Reumatología, Advance online publication. https://doi.org/10.1016/j.rcreu.2021.02.002

Wang, C., Deng, R., Gou, L., Fu, Z., Zhang, X., Shao, F., Wang, G., Fu, W., Xiao, J., Ding, X., Li, T., Xiao, X. \& Li, C. (2020). Estudio preliminar para identificar casos graves de moderados de COVID-19 utilizando parámetros hematológicos combinados. Annals of Translational Medicine , 8 (9): 593. https://doi.org/10.21037/atm-20$\underline{3391}$

Weiss, G., Ganz, T., \& Goodnough, L. T. (2019). Anemia of inflammation. Blood, 133(1): 40-50. https://doi.org/10.1182/blood-2018-06-856500 
Wu, J.T., Leung, K. \& Leung, G.M. (2020). Nowcasting and forecasting the potential domestic and international spread of the 2019-nCoV outbreak originating in Wuhan, China: a modelling study. Lancet.395:689-697. https://doi.org/10.1016/S0140-6736(20)30260-9

Wu, Z., \& McGoogan, J. M. (2020). Characteristics of and Important Lessons From the Coronavirus Disease 2019 (COVID-19) Outbreak in China: Summary of a Report of 72314 Cases From the Chinese Center for Disease Control and Prevention. JAMA, 323(13): 1239-1242. https://doi.org/10.1001/jama.2020.2648

Yang, J., Zheng, Y., Gou, X., Pu, K., Chen, Z., Guo, Q., Ji, R., Wang, H., Wang, Y., \& Zhou, Y. (2020). Prevalence of comorbidities and its effects in patients infected with SARS-CoV-2: a systematic review and metaanalysis. International journal of infectious diseases : IJID : official publication of the International Society for Infectious Diseases, 94: 91-95. https://doi.org/10.1016/j.ijid.2020.03.017

Young, B.E., Ong, S.W.X., Kalimuddin, S., Low, J.G., Tan, S.Y. \& Loh, J. (2020)- Epidemiologic Features and Clinical Course of Patients Infected With SARS-CoV-2 in Singapore. JAMA. 21;323(15):1488-1494. https://doi.org/10.1001/jama.2020.3204

Zhang, J., Wang, X., Jia, X., Li, J., Hu, K., Chen, G., Wei, J., Gong, Z., Zhou, C., Yu, H., Yu, M., Lei, H., Cheng, F., Zhang, B., Xu, Y., Wang, G., \& Dong, W. (2020). Risk factors for disease severity, unimprovement, and mortality in COVID-19 patients in Wuhan, China. Clinical microbiology and infection : the official publication of the European Society of Clinical Microbiology and Infectious Diseases, 26(6): $767-772$. https://doi.org/10.1016/j.cmi.2020.04.012 